\title{
GENERALIZED REASONING ABOUT FAULTS BASED ON THE DIAGNOSTIC MATRIX
}

\author{
MICHAŁ BARTYŚ \\ Institute of Automatic Control and Robotics \\ Warsaw University of Technology, św. A. Boboli 8, 02-525 Warsaw, Poland \\ e-mail: bartys@mchtr.pw.edu.pl
}

\begin{abstract}
This paper introduces a set of comprehensive general reasoning rules about single faults based on a diagnostic matrix. The reasoning scheme unifies inference about faults based on a conventional binary diagnostic matrix, a two- and three-valued fault isolation system as well as on their fuzzy counterparts. There are introduced and defined notions of alternative and dominant fault signatures, fuzzy fault signatures as well as a matrix of alternative signatures. This matrix is supposed to be used instead of the classic diagnostic one. It is also shown that dominant fault signatures are transformable into alternative ones. Finally, three variants of concise general reasoning rules of faults are given. Three examples illustrate key point issues of the paper. The first example refers to a medical diagnostic case. It shows an instance of dominant fault signatures and, in fact, proposes a rational approach for planning diagnostic tests. The other examples describe the fuzzy reasoning approach employing a matrix of fuzzy alternative signatures applicable for use with multi-valued fuzzy diagnostic signals. Future works are outlined in the summary section.
\end{abstract}

Keywords: fault isolation, binary diagnostic matrix, fault information system, alternative fault signature, dominant fault signature, matrix of alternative fault signatures, fuzzy diagnosis.

\section{Introduction}

Principally, model based diagnostics searches for, and afterwards makes profits from, relations between faults and residuals or their derivatives. Hence, model based diagnostics is focused on seeking for those relations, generation of residuals by means of modelling, residual processing and inferring about faults. This paper deals with the process of inferring about faults. Modelling and residual generation approaches are principally beyond the scope of this paper. Essentially, this paper contributes to a generalised reasoning scheme about faults based on the diagnostic matrix under assumption of the occurrence of single faults.

The main objective of this paper is an attempt to give a concise general description of reasoning about faults based on four known Fault Isolation (FI) approaches, namely, the structure matrix (Gertler, 1998) called further the Binary Diagnostic Matrix (BDM), the multi-valued Fault Information System (FIS) (Kościelny and Bartyś, 2000), and their fuzzy counterparts, i.e., the fuzzy diagnostic matrix (Kościelny and Bartyś, 2003; Syfert, 2006) and the Fuzzy Fault Information System (F-FIS)
(Kościelny et al., 1999; Kościelny and Syfert, 2006).

In fact, all seemingly different FI approaches stated above are based on the process of inverse causal reasoning. This implies that one may try to search for a sufficiently general reasoning scheme that matches all of them. In this context, the right choice seems to be rule based fuzzy reasoning because of its inherent ability of handling uncertain information. Formulas introduced in this paper apply under the following two restrictions:

(i) the systems considered are exclusively with single faults;

(ii) exclusively parallel reasoning scheme is considered.

The explanation of the above stated assumptions is given below. For many years, researchers have tackled the problems of detection and isolation of single and multiple faults. The results have been published in many papers, surveys and books (Frank, 1990; Gertler, 1997; 1998; Patton et al., 2000; Venkatasubramanian et al., 2003; Blanke et al., 2003; Korbicz et al., 2004; Isermann, 2006; Korbicz and Kościelny, 2010, Chen and Patton, 2012). The majority of the known Fault Detection and Isolation 
(FDI) methods were developed under the assumption of appearance of single faults. This assumption introduces a significant simplification in fault isolation. To a certain extent, the assumption (i) is acceptable because the probability of occurrence of a single fault is much higher than probability of occurrence of multiple faults. Parallel reasoning is commonly applied in theory and practice of FI (Korbicz et al., 2004, Chapter 3; Korbicz and Kościelny, 2010, Chapter 5). In some cases, it is reasonable to apply the serial reasoning scheme about faults. But it is easy to show that serial reasoning is, in fact, a sequence of parallel reasoning processes triggered by successively available symptoms of the fault. In this case, the assumption (ii) is acceptable.

The notation of fault signatures for the parallel reasoning scheme was presented indirectly by Syfert (2006) in the context of reasoning about faults based on a set of conditional statements. In his paper, Syfert did not drill out this issue further. Kościelny and Syfert (2006) introduced and defined a complex fault signature as well as reasoning about faults based on complex diagnostic rules. In fact, reasoning schemes described in the above-mentioned papers are equivalent.

We present here a slightly different approach. Firstly, a new definition of alternative fault signatures is given. In fact, this definition is also equivalent to those presented by Syfert (2006) as well as Kościelny and Syfert (2006). Secondly, we introduce the definition of dominant fault signatures. No references are known to the author in this area. Introduction of dominant fault signatures allows designing new general unified fuzzy inferring approaches applicable for process and biomedical diagnostics. Thirdly, we benefit from the statement that fuzzy reasoning is advantageous over the classical one because of its inherent generalisation features. In this context, we prove that classical approaches are to a certain extent particular cases of fuzzy reasoning. Moreover, fuzzy approaches give the opportunity of easy handling of uncertainties of diagnostic signal values and/or rules (Kościelny and Syfert, 2006).

The paper is structured as follows. A brief overview of diagnostic matrix approaches is presented in Section 2. The definition of an alternative fault signature as well as the definition of a diagnostic matrix of alternative signatures are introduced in Section 3. This section is recapitulated with an important conclusion about generality features of the diagnostic matrix of alternative signatures in the sense that it may be used for representation of any binary diagnostic matrix or multi-valued FIS. Section 4 introduces an original definition to dominant fault signatures and brings an important final conclusion about signature equivalence stating that any dominant fault signature is transformable to a set of alternative signatures.

Additionally, we propose three alternative general reasoning approaches about faults in mixed, dominant and alternative forms supported by the presentation of an appropriate medical example. Section 5 introduces the definition of fuzzy diagnosis and a substantial statement that crisp diagnosis is a particular case of fuzzy diagnosis. This describes fuzzy diagnosing as a more general approach than crispy counterparts. The fuzzy alternative fault signature defined in this section as well as the conformity degree of the actual values of fuzzy diagnostic signals with the alternative fuzzy diagnostic fault signature sets fundamentals for generalising and reformulating the fault isolation process towards fuzzy terms. A technical example is also presented. A short summary is presented in Section 6 .

\section{Diagnostic matrix approaches in FI}

A fundamental issue with reasoning about faults is the knowledge of the relation $R_{F S}$ (1) between set of faults $f_{i} \in F, \forall i \in\{1, \ldots, n\}$ and a set of diagnostic signals $s_{j} \in S, \forall j \in\{1, \ldots, m\}$ (Korbicz et al., 2004, Chapter 2). Diagnostic signals are derived from residuals by the application of appropriate evaluation processing approaches. The problem of acquisition of this knowledge, as well as methods of acquisition and evaluation of diagnostic signals, will not be addressed here. However, for simplicity, assume that the relation $R_{F S}$ is known. The relation $R_{F S}$ is defined as the Cartesian product of the sets $F$ and $S$,

$$
R_{F S} \subset F \times S \text {. }
$$

Hence, the relation $R_{F S}$ is indeed a set of $n \cdot m$ ordered pairs (bi-element relations) $\left\langle f_{i}, s_{j}\right\rangle$. According to the geometrical interpretation of the Cartesian product, the relation is the set of $n \cdot m$ points of the plane defined in the $F$ and $S$ coordinates. It is possible to spread out a three dimensional mesh over the $F \times S$ plane by attributing diagnostic signal values $v_{j, i}$ of all diagnostic signals $s_{j}$ for all $f_{i}$ faults.

The three-dimensional mesh is easily transformable to the form of a two-dimensional $m \cdot n$ matrix $V$ of the diagnostic signal values $v_{j, i}$. Hence, $V=\left[v_{j, i}\right]_{m \times n}$. In the simplest case, the diagnostic signals are two-valued, i.e., the relation faults-symptoms may have the form of a binary diagnostic matrix (Gertler, 1998; Kościelny, 2001). In this case, the set of values of diagnostic signals includes only two elements: $v=\{0,1\}, v_{j, i} \in\{v\}$. Multi-valued and fuzzy residual evaluation with a constant or adaptive threshold (Patton et al., 1989; Korbicz et al., 2004) is also used for evaluation of residuals. As results from (1), each fault $f_{i} \in F ; \forall i \in\{1, \ldots, n\}$ is associated with one and only one $i$-th column $V_{i}$ of a binary diagnostic matrix $V$

$$
V_{i}=\left[v_{1, i}, v_{2, i}, \ldots, v_{j, i}\right]^{T} .
$$


The characteristic vector $V_{i}$ containing diagnostic signal values associated with the particular fault is called the $i$-th fault signature. For notational simplicity, the diagnostic matrix $V$ will be further comprehensively represented in this paper as a block matrix of fault signatures $V_{i}$,

$$
V=\left[V_{i}\right]_{n \times 1} .
$$

An example of a binary diagnostic matrix is shown in Table 1, where five signatures are attributed to five faults $f_{1}$ to $f_{5}$. Each signature is interpreted as a specific pattern of diagnostic signals values attributed to each fault. Hence, it is profitable for reasoning about faults if those patterns are unique. However, it should be noted that, in practice, this condition typically does not necessarily hold (see, e.g., the signature of faults $f_{2}$ and $f_{3}$ in Table 1):

The following fault signatures are obtained from Table 1

$$
\begin{array}{ll}
V_{1}=[0,1,1,0,0,0]^{T}, & V_{2}=[1,0,0,0,0,0]^{T}, \\
V_{3}=[1,0,0,0,0,0]^{T}, & V_{4}=[1,1,0,0,0,0]^{T}, \\
V_{5}=[0,1,0,1,1,1]^{T} . &
\end{array}
$$

Reasoning about faults by means of a binary diagnostic matrix is based on well specified approaches of parallel, serial, serial-parallel reasoning (Korbicz et al., 2004, Chapter 3). In the case of application of parallel reasoning, fault isolation relies on searching for the identity of the vector of actual values of diagnostic signals $V_{a}$ with all fault signatures $V_{i}$. For example, if the vector of diagnostic signals $V_{a}=[1,0,0,0,0,0]^{T}$, then $V_{2}=V_{a}$ and $V_{3}=V_{a}$. Hence, the actual diagnosis $D$ (set of actually isolated single faults) is the following: $D=$ $\left\{f_{2}, f_{3}\right\}$.

Both the faults are isolated but, of course, are not distinguishable. Kościelny (2001), searching for enforcement of fault distinguishability, introduced a fault information system and a Fuzzy Fault Information System (F-FIS). He noticed that evaluation of residuals may be at least three, instead of two-valued. This gave an opportunity to handle mixed multi-valued diagnostic signal values, which made this approach more flexible when compared with the classic binary diagnostic matrix approach. Kościelny proposed the use of the FIS for reasoning about faults similar to the binary diagnostic matrix, although by applying a different inference scheme. This may be explained in an example of the mixed twoand three-valued FIS shown in Table 2. In this case, $v=\{-1,0,+1\}$ for diagnostic signals $\left\{s_{1}, s_{6}\right\}$ and $v=$ $\{0,1\}$ for the remaining signals $\left\{s_{2}, s_{3}, s_{4}, s_{5}\right\}$.

If, for example, the vector of actual values of diagnostic signals $V_{a}=[+1,0,0,0,0,0]^{T}$, then only $V_{2}=V_{a}$ and $V_{3}=V_{a}$. Hence, the actual diagnosis $D=$ $\left\{f_{2}, f_{3}\right\}$. However, by a given constant set of diagnostic signals it is possible to get a better diagnosis $D=\left\{f_{2}\right\}$ if $V_{a}=[-1,0,0,0,0,0]^{T}$, i.e., the sign of the value of $v_{1}$ will change. This allows hoping, under some conditions, to achieve better fault distinguishability compared with results of reasoning achieved by an application based on the binary diagnostic matrix approach. Please note that faults $f_{2}$ and $f_{3}$ are weakly distinguishable and are conditionally distinguishable iff $v_{1}=-1$. But if $V_{a}=[+1,0,0,0,0,0]^{T}$, then faults $f_{2}$ and $f_{3}$ remain thereinafter not distinguishable.

\section{Alternative fault signature}

The parallel reasoning scheme about faults based on a binary diagnostic matrix is transformable to the set of $n$ conditional statements:

$$
\text { if }\left(\left[V_{i}\right]_{n \times 1}=\left[V_{a}\right]_{n \times 1}\right) \text { then }\left(f_{i} \in D\right) \text {. }
$$

Please note that the binary diagnostic matrix does not determine the order in which the rules will be analysed. Hence, the analysis of the set of rules given in (4) may be carried out in any order. However, it is reasonable to analyse all the rules because of the possibility of losing information on fault distinguishability. For convenient description of the transformation process of an FIS to a set of conditional statements similar to (4), firstly we define the notion of an alternative fault signature.

Definition 1. The alternative fault signature is any block matrix column containing a relevant specific pattern of all diagnostic signals values accompanying this particular fault.

This means that each fault may be associated with more than one signature. Let the cardinality of the set of all alternative signatures assigned to each $i$-th fault be $c_{i}$
Table 1. Example of a binary diagnostic matrix.

\begin{tabular}{|c|c|c|c|c|c|}
\hline$S / F$ & $f_{1}$ & $f_{2}$ & $f_{3}$ & $f_{4}$ & $f_{5}$ \\
\hline \hline$s_{1}$ & 0 & 1 & 1 & 1 & 0 \\
$s_{2}$ & 1 & 0 & 0 & 1 & 1 \\
$s_{3}$ & 1 & 0 & 0 & 0 & 0 \\
$s_{4}$ & 0 & 0 & 0 & 0 & 1 \\
$s_{5}$ & 0 & 0 & 0 & 0 & 1 \\
$s_{6}$ & 0 & 0 & 0 & 0 & 1 \\
\hline
\end{tabular}

Table 2. Example of a mixed two- and three-valued FIS.

\begin{tabular}{|c|c|c|c|c|c|}
\hline$S / F$ & $f_{1}$ & $f_{2}$ & $f_{3}$ & $f_{4}$ & $f_{5}$ \\
\hline \hline$s_{1}$ & $0,+1$ & $-1,+1$ & +1 & +1 & 0 \\
$s_{2}$ & 1 & 0 & 0 & 1 & 1 \\
$s_{3}$ & 1 & 0 & 0 & 0 & 0 \\
$s_{4}$ & 0 & 0 & 0 & 0 & 1 \\
$s_{5}$ & 0 & 0 & 0 & 0 & 1 \\
$s_{6}$ & 0 & 0 & 0 & $0,+1$ & +1 \\
\hline
\end{tabular}


and each $k$-th alternative fault signature be denoted by $\left[V_{i}^{k}\right]_{n \times 1}$. Then the reasoning scheme about faults based on an FIS may be expressed in the form of a set of $n$ conditional statements:

$$
\text { if }\left(\exists_{k=1}^{c_{i}}\left[V_{i}^{k}\right]_{n \times 1}=\left[V_{a}\right]_{n \times 1}\right) \text { then }\left(f_{i} \in D\right) \text {. }
$$

Each conditional statement (5) owns its specific complex premise consisting of $c_{i}$ alternatives of primary Boolean premises. Let us note that by substituting $c_{i}=$ 1 in (5) we obtain (4). Here, without any doubt, the reasoning rule (5) enhances the generality of reasoning compared to the rule (4). It is worth stressing that it is possible to imagine the reasoning rule (5) where the number of primary premises does not unconditionally match $c_{i}$. In fact, this means that the set of $n$ rules (5) is not always reversibly transformable to the FIS. Hence, a set of alternative fault signatures allows a more general notation for the relation $R_{F S}$ than the FIS.

Definition 2. The diagnostic matrix of alternative signatures $V_{A}$ is a block matrix consisting of all alternative fault signatures:

$$
V_{A}=\left[\ldots\left[V_{i}^{k}\right]_{n \times 1} \ldots\right]_{1 \times q}, \quad q=\sum_{i=1}^{n} c_{i},
$$

where $c_{i}$ is the number of alternative fault signatures associated with the $i$-th fault.

Conclusion 1. The FIS and the binary diagnostic matrix are special cases of a diagnostic matrix of alternative signatures. In that sense, this matrix may be understood as a form of general representation of any binary diagnostic matrix or multi-valued FIS.

An example of the transformation of an FIS depicted in Table 2 into a diagnostic matrix of alternative signatures is given in Table 3 .

\section{Dominant fault signature}

In the practice of diagnostics of complex biological organisms, in some cases and under some conditions, reasoning about faults may be successfully carried out by

Table 3. Example of transformation of the FIS from Table 2 into a diagnostic matrix of alternative signatures.

\begin{tabular}{|c|c|c|c|c|c|c|c|c|}
\hline$S / F$ & $f_{1}^{1}$ & $f_{1}^{2}$ & $f_{2}^{1}$ & $f_{2}^{2}$ & $f_{3}^{1}$ & $f_{4}^{1}$ & $f_{4}^{2}$ & $f_{5}^{1}$ \\
\hline \hline$s_{1}$ & +1 & 0 & -1 & +1 & +1 & +1 & +1 & 0 \\
$s_{2}$ & 1 & 1 & 0 & 0 & 0 & 1 & 1 & 1 \\
$s_{3}$ & 1 & 1 & 0 & 0 & 0 & 0 & 0 & 0 \\
$s_{4}$ & 0 & 0 & 0 & 0 & 0 & 0 & 0 & 1 \\
$s_{5}$ & 0 & 0 & 0 & 0 & 0 & 0 & 0 & 1 \\
$s_{6}$ & 0 & 0 & 0 & 0 & 0 & 0 & 1 & 1 \\
\hline
\end{tabular}

consideration of specifically chosen subsets of diagnostic signals. In these cases, missing values of some diagnostic signals may not influence the result of fault isolation at all. For the description of the observation stated above, we introduce the term of dominant diagnostic signals.

Definition 3. The set of dominant diagnostic signals of the fault $f_{i}$ is any minimal subset $S_{d i}^{r_{i}}$ of the set of diagnostic signals $S$ that is sufficient to unambiguously isolate this fault.

The number $r_{i}$ of subsets $S_{d i}^{r_{i}}$ is limited by cardinality of the set of all alternative signatures assigned to $i$-th fault, $r_{i} \in\left[0, \ldots, c_{i}\right]$.

All elements of each $S_{d i}^{r_{i}}$ are called dominant diagnostic signals of the $i$-th fault.

Definition 4. The dominant fault signature $V_{d i}^{r_{i}}$ of the fault $f_{i}$ is a signature containing all dominant diagnostic signal values and for which "don"t carry" values of diagnostic signals are replaced by zero values.

Let us consider an example of the binary diagnostic matrix of alternative signatures of the fault $f_{i}$ shown in Table 4. Here, $X$ denotes any value of the diagnostic signal.

The following three sets of dominant diagnostic signals and three sets of dominant signatures result from Table 4:

$$
\begin{aligned}
S_{d 1}^{1} & =\left\{s_{1}, s_{2}, s_{3}\right\}, & S_{d 1}^{2} & =\left\{s_{4}\right\}, \\
S_{d 1}^{3} & =\left\{s_{4}, s_{6}\right\}, & V_{d 1}^{1} & =[1,0,1,0,0,0]^{T}, \\
V_{d 1}^{2} & =[0,0,0,1,0,0]^{T}, & V_{d 1}^{3} & =[0,0,0,1,0,1]^{T} .
\end{aligned}
$$

Conclusion 2. Any dominant fault signature is transformable to the set of alternative signatures containing the values of all diagnostic signals.

The dominant signature $V_{d 1}^{1}=[1,0,1,0,0,0]^{T}$ from the example given above is equivalent to the set of the

Table 4. Example of a binary diagnostic matrix with dominant fault signatures.

\begin{tabular}{|c|c|c|c|c|c|}
\hline$S / F$ & $f_{1}^{1}$ & $f_{1}^{2}$ & $f_{1}^{3}$ & $f_{1}^{4}$ & $f_{1}^{5}$ \\
\hline \hline$s_{1}$ & 1 & $X$ & $X$ & 1 & 0 \\
$s_{2}$ & 0 & $X$ & $X$ & 1 & 1 \\
$s_{3}$ & 1 & $X$ & $X$ & 1 & 1 \\
$s_{4}$ & $X$ & 1 & 1 & 0 & 0 \\
$s_{5}$ & $X$ & $X$ & $X$ & 1 & 1 \\
$s_{6}$ & $X$ & $X$ & 1 & 1 & 1 \\
\hline
\end{tabular}


following eight alternative signatures:

$$
\begin{array}{lll}
V_{1}^{1}=[1,0,1,0,0,0]^{T}, & V_{1}^{2}=[1,0,1,0,0,1]^{T}, \\
V_{1}^{3}=[1,0,1,0,1,0]^{T}, & V_{1}^{4}=[1,0,1,0,1,1]^{T}, \\
V_{1}^{5}=[1,0,1,1,0,0]^{T}, & V_{1}^{6}=[1,0,1,1,0,1]^{T}, \\
V_{1}^{7}=[1,0,1,1,1,0]^{T}, & V_{1}^{8}=[1,0,1,1,1,1]^{T} .
\end{array}
$$

Dominant signatures allow us to use a concise notation of alternative signatures. But, in fact, the knowledge about dominant signatures is in practice much more important. This will be thereinafter explained. For each set $S_{d i}$, let us design an $m$-element characteristic set $\Psi_{d i}$, where the binary elements equal to 1 are assigned to all the elements from the set $S$ that belong to the set of dominant diagnostic signals, and the binary values 0 are assigned to all the remaining elements. Hence, for each set of dominant diagnostic signals of the fault $f_{i}$, the following holds:

$$
S_{d i}=\left\{\left(s_{j} \in S: \psi_{d i, j}=1\right)\right\}, \quad \forall j \in\{1, \ldots, m\},
$$

and

$$
\Psi_{d i}=\left\{\psi_{d i, j}\right\}, \quad \forall j \in\{1, \ldots, m\} .
$$

Let us now define alternative and dominant premises.

Definition 5. The alternative premise $p_{a i}$ of the conditional statement reasoning about fault $f_{i}$ is a Boolean alternative of comparisons results of all sets of alternative fault signatures $\left[V_{i}^{k}\right]_{n \times 1}$ with the set of actual values of diagnostic signals $\left[V_{a}\right]_{n \times 1}$,

$$
p_{a i}=\bigcup_{k=1}^{c_{i}} p_{a i}^{k}
$$

where

$$
p_{a i}^{k}=\left\{\begin{array}{lll}
0 & \text { if } \quad\left[V_{i}^{k}\right]_{n \times 1} \neq\left[V_{a}\right]_{n \times 1}, \\
1 & \text { if } \quad\left[V_{i}^{k}\right]_{n \times 1}=\left[V_{a}\right]_{n \times 1}
\end{array}\right.
$$

Definition 6. A dominant premise $p_{d i}$ of the conditional statement reasoning about fault $f_{i}$ is a Boolean alternative of all the results of comparison of the dominant fault signature, with all $l$ sets of actual values of dominant diagnostic signals $\left[V_{a}^{l}\right]_{n \times 1}=\left[V_{a}\right]_{n \times 1} \cap\left[V_{d i}^{l}\right]_{n \times 1}$,

$$
p_{d i}=\bigcup_{l=1}^{l_{i}} p_{d i}^{l}
$$

where $l_{i}$ is a count of dominant signatures of the fault $f_{i}$ and

$$
p_{d i}^{l}=\left\{\begin{array}{lll}
0 & \text { if } & {\left[V_{d i}^{l}\right]_{n \times 1} \neq\left[V_{a}^{l}\right]_{n \times 1},} \\
1 & \text { if } \quad\left[V_{d i}^{l}\right]_{n \times 1}=\left[V_{a}^{l}\right]_{n \times 1} .
\end{array}\right.
$$

Lemma 1. The dominant and alternative premises are equivalent.
Proof. By definition, each dominant fault signature is transformable to a set of alternative signatures. But each alternative fault signature is simultaneously a dominant one. Hence, dominant and alternative premises are equivalent.

Let us come back to the example given above. We have the dominant signature $V_{d 1}^{1}=[1,0,1,0,0,0]^{T}$ and a set of actual diagnostic values equal to $V_{a}=$ $[1,0,1,1,0,1]^{T}$. The dominant signature $V_{d 1}^{1}$ may be transformed to the set of eight alternative signatures. Hence, in the worst case, true value of the alternative premise may be found after eight comparisons. It might also be found earlier. Let us assume that the mean value of the number of comparisons equals 4 . In the same example, the true value of the dominant premise is found after only two operations: a modification of the set of actual values of diagnostic signals and one comparison. Here, the number of operations does not depend on values of the signatures. Taking into account the substitutions (9), (11) and Lemma 1, the rule (5) will be rewritten in the following three alternative general comprehensive forms:

(i) unified mixed form,

$$
\text { if }\left(p_{d i} \vee p_{a i}\right) \text { then }\left(f_{i} \in D\right) \text {; }
$$

(ii) unified dominant form,

$$
\text { if }\left(p_{d i}\right) \text { then }\left(f_{i} \in D\right) \text {; }
$$

(iii) unified alternative form,

$$
\text { if }\left(p_{a i}\right) \text { then }\left(f_{i} \in D\right) .
$$

The rule (13) summarizes parallel reasoning about faults as a process of searching for the true value of either dominant or alternative fault premises. The rule (13) has significant practical meaning. It allows feasible planning of sequences of diagnostic tests in off-line mode diagnostics. It is reasonable to perform diagnostic tests of the diagnostic signals belonging to the sets of dominant fault signatures in the first instance.

Example 1. Consider the diagnostics of a carcinoma renal case. Carcinoma renal is a kidney cancer responsible for about $80 \%$ of cases. The majority of renal cancers are asymptomatic. This implies consideration of rich sets of alternative signatures in the diagnostic process, which makes unambiguous diagnosis hard. Hopefully, for kidney diagnostics, in $10-15 \%$ of cases, a set of three symptoms: kidney tumour, kidney pain and haematuria, is generally indicative in more advanced states of the disease. These three symptoms (triad) play the dominant role in kidney cancer diagnostics. Therefore, this triad of symptoms conditionally forms a dominant signature of the kidney cancer disease. 
Of course, it is not reasonable to assume that this particular dominant triad is specific only and only for the carcinoma renal case. The same triad may be revealed in different diseases and it may also play a dominating role. In such cases, dominant signatures may be alternative as well, and the mixed form (13) of reasoning about faults is recommended for applications.

An extract of the diagnostic matrix of alternative signatures of carcinoma renal is shown in Table 5. Here, the first dominant signature is represented by the set of diagnostic signals $\left\{s_{1}, s_{2}, s_{3}\right\}$. An examination of a tissue specimen from a biopsy is considered essentially in oncology for proper identification of cancer. Hence, the result of histopathology examination of kidney tumour tissue forms the next dominant signature. It is represented in Table 5 by the single set of diagnostic signals $\left\{s_{4}\right\}$. Sometimes biopsy is not possible or is ordered as a secondary diagnostic test to confirm suspicions of cancer disease.

As can be seen from Table 5, there are two sets $\left(l_{1}=\right.$ 2 ) of dominant diagnostic signals $S_{d 1}^{1}$ and $S_{d 1}^{2}$ :

$$
S_{d 1}^{1}=\left\{s_{1}, s_{2}, s_{3}\right\}, \quad S_{d 1}^{2}=\left\{s_{4}\right\} .
$$

From (7) and the definition (4), we deduce the following dominant signatures of the fault $f_{1}$ :

$$
\begin{aligned}
V_{d 1}^{1} & =[1,1,1,0,0,0,0,0,0,0,0,0,0,0,0,0]_{16 \times 1}^{T}, \\
V_{d 1}^{2} & =[0,0,0,1,0,0,0,0,0,0,0,0,0,0,0,0]_{16 \times 1}^{T} .
\end{aligned}
$$

Assume that at time instant $t_{0}$ there are results available of five diagnostic tests only:

$$
\begin{aligned}
& V_{a}^{t_{0}} \\
= & {[1,1,1,-, 0,-,-,-, 1,-,-,-,-,-,-,-]_{16 \times 1}^{T} . }
\end{aligned}
$$

Table 5. Extract of a diagnostic matrix that exemplify diagnostics of the carcinoma renal case ( $f_{1}^{c_{1}}$ : carcinoma renal, $X$ : "don't carry", lack of diagnostic test).

\begin{tabular}{|c|c|c|c|c|c|c|}
\hline$S / F$ & $f_{1}^{1}$ & $f_{1}^{2}$ & $f_{1}^{3}$ & $\ldots$ & $f_{1}^{c_{1}}$ & Diagnostic tests \\
\hline \hline$s_{1}$ & 1 & $X$ & 1 & $\ldots$ & 0 & kidney tumour \\
$s_{2}$ & 1 & $X$ & 0 & $\ldots$ & 0 & kidney pain \\
$s_{3}$ & 1 & $X$ & 1 & $\ldots$ & 0 & haematuria \\
$s_{4}$ & $X$ & 1 & - & $\ldots$ & - & histopathology \\
$s_{5}$ & $X$ & $X$ & 1 & $\ldots$ & 1 & weight loss \\
$s_{6}$ & $X$ & $X$ & 1 & $\ldots$ & 1 & polycythaemia \\
$s_{7}$ & $X$ & $X$ & 0 & $\ldots$ & 0 & anaemia \\
$s_{8}$ & $X$ & $X$ & 1 & $\ldots$ & 1 & hypercalcaemia \\
$s_{9}$ & $X$ & $X$ & 0 & $\ldots$ & 1 & night sweats \\
$s_{10}$ & $X$ & $X$ & 1 & $\ldots$ & 1 & hypertension \\
$s_{11}$ & $X$ & $X$ & 1 & $\ldots$ & 1 & high ESR \\
$s_{12}$ & $X$ & $X$ & 1 & $\ldots$ & 1 & myositis \\
$s_{13}$ & $X$ & $X$ & 1 & $\ldots$ & 1 & amyloidosis \\
$s_{14}$ & $X$ & $X$ & 1 & $\ldots$ & 1 & creatinine \\
$s_{15}$ & $X$ & $X$ & 0 & $\ldots$ & 1 & leg swelling \\
$s_{16}$ & $X$ & $X$ & 0 & $\ldots$ & 1 & hyperthermia \\
\hline
\end{tabular}

Is it possible in this situation to infer about the carcinoma renal case? In the classical dynamic reasoning approach, it is possible to do this. But in this reasoning case, the lack of some diagnostic signals generally impairs fault distinguishability. If there are defined dominant signatures, inferring about the carcinoma renal case is recommended at each stage of the acquisition of diagnostic signals. The fault is ambiguously isolated and the process of fault isolation may be stopped immediately after achieving the value of dominant signature equal to 1 .

Please note that the value of the dominant premise in (13) or (14) equals 1 for a given set of dominant signatures (17) and a set of available results of diagnostic tests $V_{a}^{t_{0}}$,

$$
p_{d 1}=1 \vee 0=1 \text {. }
$$

If, for example, at time instant $t_{1}$, the set of diagnostic signals is

$$
\begin{aligned}
& V_{a}^{t_{1}} \\
= & {[1,1,-,-, 0,-,-,-, 1,0,-,-,-,-,-,-]_{16 \times 1}^{T}, }
\end{aligned}
$$

then the value of the dominant premise is $p_{d 1}=0$ and process of diagnostics of the carcinoma renal case should be continued.

\section{Fuzzy fault signatures}

In practice, results of evaluation of diagnostic signals are more or less uncertain. This is caused by many factors such as measurement errors, measuring noise, application of imprecise models for residual generation, etc. This, in fact, should be addressed in reasoning about faults. Reasoning about faults taking into account uncertain symptoms was discussed by Syfert (2006), Kościelny et al. (1999) as well as Korbicz et al. (2004), and found many applications in process industries, e.g., in diagnostics of a sugar juice evaporation station, the steam-water line of a boiler in a power station, or a pneumatic actuator-positioner control valve assembly.

It is clear that rules (13)-(15) are not general enough to be used directly for description of fuzzy reasoning about faults. Below it will be shown that these rules, after appropriate modifications, may be applied to fuzzy reasoning, too. Firstly, let us assign the binary characteristic function $\Phi$ of the diagnosis set $D$ that assigns each fault $f_{i} \in F$ a Boolean value $\phi_{i}$ :

$$
\left\{\begin{aligned}
\phi_{i} & =0 \Leftrightarrow f_{i} \notin D, \\
\phi_{i} & =1 \Leftrightarrow f_{i} \in D .
\end{aligned}\right.
$$

The introduction of the characteristic function allows us to reformulate the general conditional reasoning statement (15) to its alternative form (20) convenient for further discussion,

$$
\text { if }\left(p_{a i}=1\right) \text { then } \quad\left(\phi_{i}=1\right) .
$$


Here, the Boolean premise reflects an alternative of all results of comparison of the set of actual fault diagnostic signals with all alternative fault signatures.

Definition 7. The fuzzy diagnosis $D^{F}$ is a fuzzy set defined on a space of faults $D^{F} \subseteq F$. Because cardinality of the set of faults $F$ is equal to $n$, the fuzzy diagnosis is the set of $n$ pairs,

$$
D^{F}=\bigvee_{i=1}^{n}\left\langle\mu_{i} / f_{i}\right\rangle, \quad \forall f_{i} \in F,
$$

where $\mu_{i} \in[0,1]$.

Let us transform (20) to a fuzzy reasoning rule about the fault $f_{i}$ based on Mamdani's implication:

$$
\text { if }\left(p_{a i}^{F}=\tau_{i}\right) \text { then }\left(\mu_{i}=\tau_{i}\right),
$$

where $p_{a i}^{F}$ is the fuzzy alternative premise of fault $f_{i} \in F$, $\tau_{i}$ is the fuzzy rule activation level $\tau_{i} \in[0,1], \mu_{i}$ is the membership function of the fuzzy diagnosis.

Conclusion 3. A crisp diagnosis $D$ is a particular case of a fuzzy diagnosis $D^{F}$ for which $\tau_{i} \in[0,1]$. In that sense, fuzzy diagnosis about faults is more general. Calculation of fuzzy alternative premises is a separate problem in (Korbicz et al., 2004, Chapter 11) beyond the scope of this paper.

Example 2. Let us consider a multi-valued fault information system with crisp fault signatures as shown in Table 2. Now, let each residual be fuzzy evaluated by means of specific symmetric fuzzy sets with the exception that for evaluation of two-valued residuals the absolute value of the residual is used instead of its signed value.

Definition 8. The fuzzy diagnostic signal $s^{F}$ is a $p$-valued linguistic variable defined in the residual space of discourse.

Remark 1. The general reasoning rule (22) formally does not make restrictions on the value of $p$.

Let fuzzy diagnostic signals sets be defined by $\Pi$ shaped membership functions as depicted in Fig. 1. For simplicity, all appropriate fuzzy values (fuzzy sets) of all fuzzy diagnostic signals in this example are identical.

Definition 9. The fuzzy diagnostic signal value $v^{F}$ of a fuzzy diagnostic signal $s^{F}$ is a $p$-tuple value of the membership functions values $\left\langle\mu_{1}, \mu_{2}, \ldots, \mu_{p}\right\rangle$ assigned to the particular residual value $r_{j}$.

Let us assume that at time instant $t$ the following set of normalised residuals was obtained: $R_{a}=$ $\{0.7,1.0,-0.4,0.3,0.5,-0.1\}$. In part, the problem of normalization of residuals was tackled by Bartyś et al.
(2005). Fuzzy values of these residuals (actual fuzzy diagnostic values $v_{a j}^{F}$ ) are shown in Table 6. For example, if $v_{a 1}^{F}=\langle 0.0,0.1,0.9\rangle$ then normalised residual $r_{a 1}=0.7$ is considered non-negative (degree $=0.0$ ), in part close to zero $($ degree $=0.1)$, and in some part positive $($ degree $=$ $0.9)$.

Definition 10. The fuzzy alternative fault signature $S^{F}$ is an $m$-valued linguistic variable defined in the space of discourse being the values of fuzzy diagnostic signals $S$ that contain a relevant specific pattern of all the fuzzy diagnostic signal values accompanying the occurrence of the particular fault.

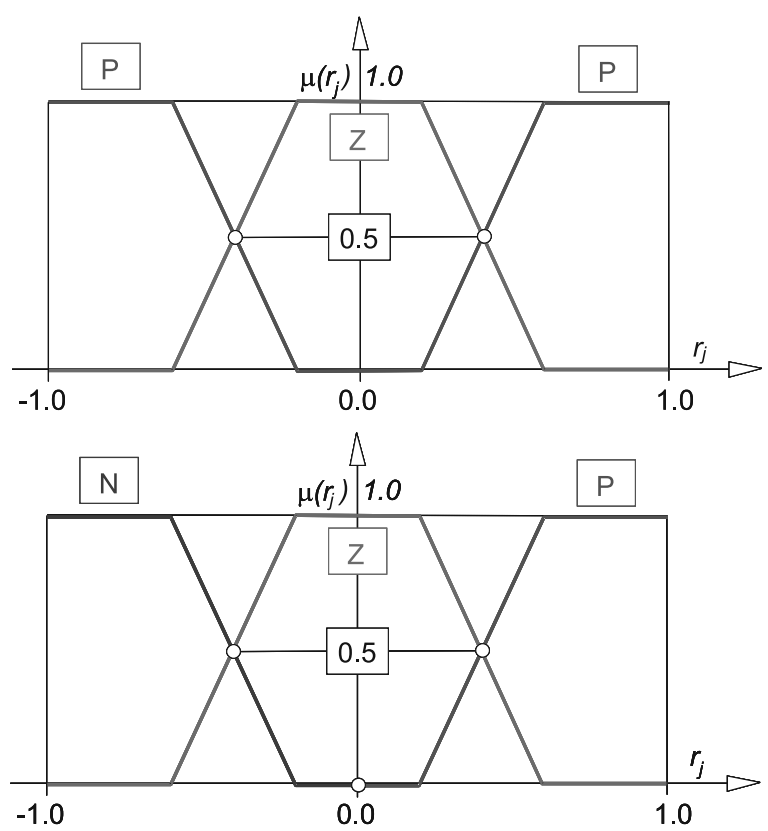

Fig. 1. Example of two- and three-valued fuzzy evaluation of the $j$-th residual $\left(r_{j}\right.$ : normalised value of the $j$-th residual, $N, Z, P$ : fuzzy values of the linguistic variable $r_{j}$ ).

Let fuzzy alternative fault signatures of all faults be known (Table 7). For example, in Table 7 there are two alternative fuzzy signatures $S_{1,1}^{F}=\{P, P, P, Z, Z, Z\}$ and $S_{1,2}^{F}=\{Z, P, P, Z, Z, Z\}$ of fault $f_{1}$ and only one alternative fuzzy signature $S_{5,1}^{F}=\{Z, P, Z, P, P, P\}$ of fault $f_{5}$.

Let us now create Table 8 as a copy of Table 7 , in which symbols of fuzzy alternative fault signatures $S^{F}$ from Table 7 are replaced appropriately by the values of actual fuzzy diagnostic signals from Table 6. Additionally, we define the following three candidate measures of the conformity degrees $\tau_{i, k}$ of the actual values of fuzzy diagnostic signals $V_{a}^{F}$ with the $k$-th alternative fuzzy diagnostic fault signature of fault $f_{i}$ in 
Table 6. Actual fuzzy diagnostic signals values of a multivalued fuzzy fault information system.

\begin{tabular}{|c|c|c|c|r|r|r|}
\hline$V^{F} / s^{F}$ & $N$ & $Z$ & $P$ & & $R_{a}$ & $p$ \\
\hline \hline$v_{a 1}^{F}$ & 0.0 & 0.1 & 0.9 & & 0.7 & 3 \\
$v_{a 2}^{F}$ & 0.0 & 0.0 & 1.0 & & 1.0 & 2 \\
$v_{a 3}^{F}$ & 0.0 & 0.7 & 0.3 & & -0.32 & 2 \\
$v_{a 4}^{F}$ & 0.0 & 0.9 & 0.1 & & 0.3 & 2 \\
$v_{a 5}^{F}$ & 0.0 & 0.5 & 0.5 & & 0.5 & 2 \\
$v_{a 6}^{F}$ & 0.0 & 1.0 & 0.0 & & -0.1 & 3 \\
\hline
\end{tabular}

Table 7. Fuzzy alternative fault signatures of a multi-valued fuzzy fault information system.

\begin{tabular}{|c|c|c|c|c|c|c|c|c|}
\hline$S^{F} / F$ & $f_{1}^{1}$ & $f_{1}^{2}$ & $f_{2}^{1}$ & $f_{2}^{2}$ & $f_{3}^{1}$ & $f_{4}^{1}$ & $f_{4}^{2}$ & $f_{5}^{1}$ \\
\hline \hline$s_{1}^{F}$ & $P$ & $Z$ & $N$ & $P$ & $P$ & $P$ & $P$ & $Z$ \\
$s_{2}^{F}$ & $P$ & $P$ & $Z$ & $Z$ & $Z$ & $P$ & $P$ & $P$ \\
$s_{3}^{F}$ & $P$ & $P$ & $Z$ & $Z$ & $Z$ & $Z$ & $Z$ & $Z$ \\
$s_{4}^{F}$ & $Z$ & $Z$ & $Z$ & $Z$ & $Z$ & $Z$ & $Z$ & $P$ \\
$s_{5}^{F}$ & $Z$ & $Z$ & $Z$ & $Z$ & $Z$ & $Z$ & $Z$ & $P$ \\
$s_{6}^{F}$ & $Z$ & $Z$ & $Z$ & $Z$ & $Z$ & $P$ & $P$ & $P$ \\
\hline
\end{tabular}

(i) mean value,

$$
\tau_{i, k}=\frac{1}{m} \sum_{j=1}^{m} s_{i, k, j}^{F}
$$

(ii) product value,

$$
\tau_{i, k}=\prod_{j=1}^{m} s_{i, k, j}^{F}
$$

(iii) minimum value,

$$
\tau_{i, k}=\bigwedge_{j=1}^{m} s_{i, k, j}^{F} .
$$

Let the conformity degree $\tau_{i}$ of the $i$-th fault of the actual values of fuzzy diagnostic signals $V_{a}^{F}$ with all fuzzy diagnostic fault signatures of fault $f_{i}$ be defined as the maximum of $\tau_{i, k}$ values:

$$
\tau_{i}=\bigvee_{k=1}^{c_{i, k}} \tau_{i, k}
$$

where $c_{i, k}$ is the number of the alternative fault signatures associated with the $i$-th fault.

In fact, $\tau_{i}$ is an activation level of the fuzzy rule (22) inferring about fault $f_{i}$. Call this level the certainty degree the $i$-th fault.

The mean value (23) of the conformity degrees $\tau_{i, k}$ is characterised by low-pass features. This is beneficial in real applications because it introduces to a certain extent immunity against spurious diagnoses due to undesired disturbances in actual values of diagnostic signals. On the other hand, it flattens the conformity degrees of the faults, which can be easily seen from Table 8 .

The fuzzy diagnosis $D^{F}$ obtained for a given set of actual residual values $R_{a}$ from Table 6 by application of a measure of conformity (23) is

$$
D^{F}=\left\{0.60 / f_{1}, 0.83 / f_{2}, 0.83 / f_{3}, 0.77 / f_{4}, 0.33 / f_{5}\right\} .
$$

This diagnosis indicates all isolated faults assigning to them some factors that may be interpreted as similarity degrees of the fuzzy fault signatures to the fuzzy actual values of diagnostic signals. By arbitrarily choosing some acceptable similarity threshold value $T$, it is possible to highlight most certain faults. In our case, if we choose the similarity threshold $T=0.70$, then

$$
D_{T=0.7}^{F}=\left\{0.83 / f_{2}, 0.83 / f_{3}, 0.77 / f_{4}\right\} .
$$

Applying (24) and (25), we obtain respectively

$$
\begin{aligned}
D_{\mathrm{PROD}}^{F} & =\left\{0.28 / f_{2}, 0.28 / f_{3}, 0.12 / f_{4}\right\}, \\
D_{\mathrm{MIN}}^{F} & =\left\{0.50 / f_{2}, 0.50 / f_{3}, 0.30 / f_{4}\right\} .
\end{aligned}
$$

Basically all the three examined measures of conformity bring similar diagnoses in the theoretical example considered. Let us examine now a more practical example.

Example 3. Consider now an intelligent electro-pneumatic final control element (Fig. 2), nowadays commonly used in industrial practice. This element is an assembly consisting of the following three main components: a positioner, a spring-and-diaphragm pneumatic servomotor, and a control valve. The control valve acts on the flow of the fluid passing through the pipeline installation. The pneumatic actuator carries out a change in the position of the control valve plug, thus acting on a fluid flow rate. The spring-and-diaphragm pneumatic servomotor is an air driven device in which the compressed air produced by the electro-pneumatic transducer $E / P$ acts upon the flexible diaphragm to provide a linear motion $X$ of the servomotor stem. The positioner is a device applied to control valve stem positions.

Table 8. Fuzzy alternative fault conformity degrees of a multivalued fuzzy fault information system.

\begin{tabular}{|c|c|c|c|c|c|c|c|c|}
\hline$S^{F} / F$ & $f_{1}^{1}$ & $f_{1}^{2}$ & $f_{2}^{1}$ & $f_{2}^{2}$ & $f_{3}^{1}$ & $f_{4}^{1}$ & $f_{4}^{2}$ & $f_{5}^{1}$ \\
\hline \hline$s_{1}^{F}$ & 0.9 & 0.1 & 0.0 & 0.9 & 0.9 & 0.9 & 0.9 & 0.1 \\
$s_{2}^{F}$ & 0.3 & 0.3 & 0.7 & 0.7 & 0.7 & 0.3 & 0.3 & 0.3 \\
$s_{3}^{F}$ & 0.0 & 0.0 & 1.0 & 1.0 & 1.0 & 1.0 & 1.0 & 1.0 \\
$s_{4}^{F}$ & 0.9 & 0.9 & 0.9 & 0.9 & 0.9 & 0.9 & 0.9 & 0.1 \\
$s_{5}^{F}$ & 0.5 & 0.5 & 0.5 & 0.5 & 0.5 & 0.5 & 0.5 & 0.5 \\
$s_{6}^{F}$ & 1.0 & 1.0 & 1.0 & 1.0 & 1.0 & 1.0 & 0.0 & 0.0 \\
\hline
\end{tabular}




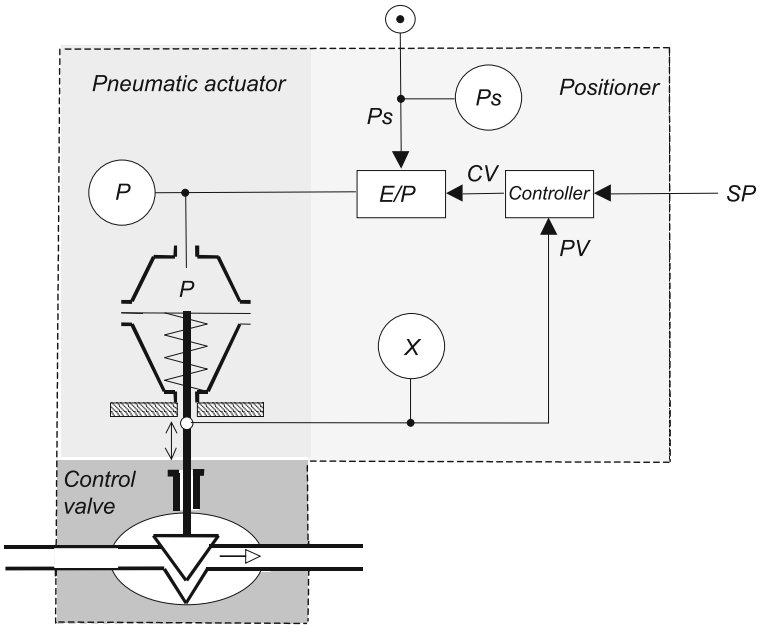

Fig. 2. Structure of the electro-pneumatic final control element considered.

The actuator may be considered a set of two subsystems. The first subsystem consists of a positioner and a pneumatic actuator. This subsystem is a closed loop system that transforms the external set point signal SP into the physical position $X$ of the stem of the pneumatic actuator. The external set point signal $S P$ is delivered from an external control system by an analogue or a digital communication link. The stem position $X$ is used as the position feedback signal for the internal controller of the positioner. The second subsystem transforms servomotor linear stem displacement $X$ into the medium flow rate $F$ according to the specific flow characteristics of the control valve. Internally, there are only three physical measurements available. In this example, we will assume that faults of all these measurements will not be considered.

When discussing the embedded diagnostics of a final control element, it is obvious that the set point value $S P$, the process value $P V$ as well as the control value $C V$ signals are available and may play the role of virtual measurements. In this example we will assume also that $S P, P V$ and $C V$ signals are fault free. This indirectly implies that positioner controller is also faultless. The list of available measurements and the list of faults in the final control element considered are respectively presented in Tables 9 and 10 .

The five faults selectively affect the final control element parts. The causal graph of the final control

Table 9. List of available measurements

\begin{tabular}{|c|l|l|}
\hline$N_{o}$ & Symbol & Description \\
\hline \hline 1 & $P_{s}$ & Air supply pressure \\
2 & $P$ & Air pressure in the chamber \\
3 & $X$ & Actuator stem displacement \\
\hline
\end{tabular}

element depicted in Fig. 3 illustrates the influence of these faults on the graph's transitions.

The set of four residuals $\left\{r_{1}, r_{2}, r_{3}, r_{4}\right\}$ shown in Table 11 is proposed for model-based fault detection purposes. Residuals are calculated based on simple soft computing partial models depicted in Table 11.

Let us apply now two- and three-valued fuzzy evaluation of residuals from Table 11 based on the fuzzy set definitions presented in Fig. 1. Prior to the explanation of fuzzy diagnosing, let us construct a fuzzy fault information system (Table 12).

Please notice that if the value of air supply pressure $P_{s}$ drops down below some critical level then the final control element switches immediately to an inoperable state despite the values of all remaining diagnostic signals. In this context, the signal $s_{1}$ plays the dominant role: $S_{d 1}^{1}=\left\{s_{1}\right\}$. Introduction of dominant diagnostic

Table 10. List of faults considered.

\begin{tabular}{|c|l|l|}
\hline Fault & Description & Type \\
\hline \hline$f_{1}$ & Supply air pressure & external \\
$f_{2}$ & E-P transducer & internal \\
$f_{3}$ & Pneumatic actuator & internal \\
$f_{4}$ & Position feedback & internal \\
$f_{5}$ & Control valve & external \\
\hline
\end{tabular}

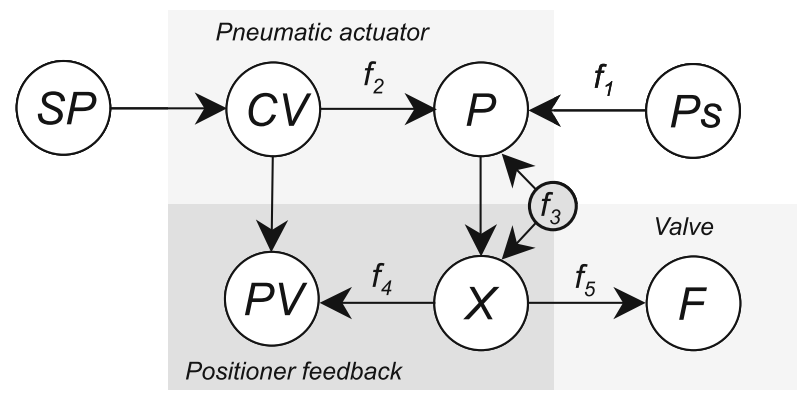

Fig. 3. Causal graph of the electro-pneumatic final control element.

Table 11. List of residuals and diagnostic signals.

\begin{tabular}{|l|l|l|}
\hline Symbol & Residual & Affected by \\
\hline \hline$s_{1}$ & $r_{1}=\widehat{P}_{s}-f\left(P_{s}\right)$ & $f_{1}$ \\
$s_{2}$ & $r_{2}=\widehat{P}-f(C V)$ & $f_{1}, f_{2}, f_{3}$ \\
$s_{3}$ & $r_{3}=\widehat{X}-f(P)$ & $f_{3}, f_{5}$ \\
$s_{4}$ & $r_{4}=\widehat{P V}-f(X)$ & $f_{4}$ \\
\hline
\end{tabular}

Table 12. Multi-valued fuzzy fault information system.

\begin{tabular}{|c|c|c|c|c|c|}
\hline$S / F$ & $f_{1}$ & $f_{2}$ & $f_{3}$ & $f_{4}$ & $f_{5}$ \\
\hline \hline$s_{1}$ & $P$ & $N$ & $N$ & $N$ & $N$ \\
$s_{2}$ & $N, P$ & $N, P$ & $Z, N$ & $Z$ & $Z$ \\
$s_{3}$ & $Z$ & $Z$ & $P$ & $Z$ & $P$ \\
$s_{4}$ & $Z$ & $Z$ & $Z$ & $P$ & $Z$ \\
\hline
\end{tabular}


signals allows us to prioritize diagnostic threads in order to optimize diagnostic process performance. This is particularly important in the case of application of an external diagnostic system. The actual membership function values of diagnostic signals of the multi-valued fuzzy fault information system is presented in Table 13.

Now we will design fuzzy alternative fault signatures (Table 14) based on Definition 9.

Next, we substitute fuzzy sets terms from Table 14 by actual membership function values of diagnostic signals from Table 13. The results are presented in Table 15 in the form of a matrix of fuzzy alternative fault conformity degrees.

Finally, the fuzzy diagnosis $D_{F}$ in the form (21) obtained for a given set of actual residual values $R_{a}$ from Table 13 by application of the measure of conformity (23) is as follows:

$$
D^{F}=\left\{0.88 / f_{1}, 0.63 / f_{2}, 0.33 / f_{3}, 0.23 / f_{4}, 0.33 / f_{5}\right\} .
$$

If we choose the same value of the similarity threshold $T=0.70$ as in Example 2, then

$$
D_{T=0.7}^{F}=\{0.88 / f 1\} .
$$

Applying (24) and (25), from Table 15 we obtain respectively

$$
\begin{aligned}
D_{\text {PROD }}^{F} & =\left\{0.57 / f_{1}\right\}, \\
D_{\text {MIN }}^{F} & =\left\{0.70 / f_{1}\right\} .
\end{aligned}
$$

Table 13. Sample actual fuzzy diagnostic signals values of a multi-valued fuzzy fault information system.

\begin{tabular}{|c|c|c|c|r|r|r|}
\hline$V^{F} / s^{F}$ & $N$ & $Z$ & $P$ & $R_{a}$ & $p$ \\
\hline \hline$v_{a 1}^{F}$ & 0.0 & 0.0 & 1.0 & & 0.9 & 2 \\
$v_{a 2}^{F}$ & 0.0 & 0.1 & 0.9 & & 0.7 & 3 \\
$v_{a 3}^{F}$ & 0.0 & 0.7 & 0.3 & & -0.32 & 2 \\
$v_{a 4}^{F}$ & 0.0 & 0.9 & 0.1 & & 0.3 & 2 \\
\hline
\end{tabular}

Table 14. Fuzzy alternative fault signatures of a fuzzy fault information system.

\begin{tabular}{|c|c|c|c|c|c|c|c|c|}
\hline$S^{F} / F$ & $f_{1}^{1}$ & $f_{1}^{2}$ & $f_{2}^{1}$ & $f_{2}^{2}$ & $f_{3}^{1}$ & $f_{4}^{1}$ & $f_{4}^{2}$ & $f_{5}^{1}$ \\
\hline \hline$s_{1}^{F}$ & $P$ & $P$ & $Z$ & $Z$ & $Z$ & $Z$ & $Z$ & $Z$ \\
$s_{2}^{F}$ & $N$ & $P$ & $N$ & $P$ & $Z$ & $N$ & $Z$ & $Z$ \\
$s_{3}^{F}$ & $Z$ & $Z$ & $Z$ & $Z$ & $P$ & $P$ & $Z$ & $P$ \\
$s_{4}^{F}$ & $Z$ & $Z$ & $Z$ & $Z$ & $Z$ & $Z$ & $P$ & $Z$ \\
\hline
\end{tabular}

Table 15. Fuzzy alternative fault conformity degrees of a multivalued fuzzy fault information system.

\begin{tabular}{|c|c|c|c|c|c|c|c|c|}
\hline$S^{F} / F$ & $f_{1}^{1}$ & $f_{1}^{2}$ & $f_{2}^{1}$ & $f_{2}^{2}$ & $f_{3}^{1}$ & $f_{3}^{2}$ & $f_{4}^{1}$ & $f_{5}^{1}$ \\
\hline \hline$s_{1}^{F}$ & 1.0 & 1.0 & 0.0 & 0.0 & 0.0 & 0.0 & 0.0 & 0.0 \\
$s_{2}^{F}$ & 0.0 & 0.9 & 0.0 & 0.9 & 0.1 & 0.0 & 0.1 & 0.1 \\
$s_{3}^{F}$ & 0.7 & 0.7 & 0.7 & 0.7 & 0.3 & 0.3 & 0.7 & 0.3 \\
$s_{4}^{F}$ & 0.9 & 0.9 & 0.9 & 0.9 & 0.9 & 0.9 & 0.1 & 0.9 \\
\hline
\end{tabular}

Also in this example, all three examined measures of conformity brought similar diagnoses. In contrast to (23), the product (24) and minimum (25) operations are sensitive to incidental zero values of diagnostic signals, which may reflect in the flickering of diagnoses. On the other hand, the necessity of an arbitrary choice of fixed or adaptive values of the trigger level is disadvantageous for this approach. Hence, if the actual values of diagnostic signals are carefully validated in the pre-processing phase prior to applying FI, then the mean value (23) of the conformity degree should not be considered in practice to be the best choice.

\section{Summary}

There were defined alternative and dominant fault signatures. It was proven that dominant fault signatures are convertible to alternative ones. Knowledge about dominant fault signatures makes it possible to speed up the reasoning process and allows rational planning of diagnostic tests in off-line diagnostics. It was proven that fuzzy reasoning about faults based on a diagnostic matrix of alternative fault signatures has better generalization features compared with its crisp counterparts.

It was shown, that fault isolation system may be decomposed into a matrix of alternative fuzzy signatures. However, inverse transformation is not always possible.

Finally, three general forms of reasoning about faults are presented: uniform alternative, uniform dominant and mixed. All forms apply to crisp and fuzzy reasoning about faults.

Future works will be focused on searching for a generalized description of reasoning about multiple faults.

\section{Acknowledgment}

This work was supported by the Polish National Science Centre within the research project DEC-2011/01/B/ST7/06183.

\section{References}

Bartyś, M., Kościelny, J.M. and Rzepiejewski, P. (2005). Fuzzy logic application for fault isolation of actuators, Computer Assisted Mechanics and Engineering Sciences 12(2-3): 89-102.

Blanke, M., Kinnaert, M., Lunze, J. and Staroswiecki, M. (2003). Diagnosis and Fault-Tolerant Control, Springer-Verlag, Berlin/Heidelberg/New York, NY.

Chen, J. and Patton, R.J. (2012). Robust Model-based Fault Diagnosis for Dynamic Systems, Springer, London.

Frank, P.M. (1990). Fault diagnosis in dynamic systems using analytical and knowledge-based redundancy-a survey and some new results, Automatica 26(3): 459-474.

Gertler, J. (1997). Fault detection and isolation using parity relations, Control Engineering Practice 5(5): 653-661. 
Gertler, J. (1998). Fault Detection and Diagnosis in Engineering Systems, Marcel Dekker Inc., New York, NY.

Isermann, R. (2006). Fault Diagnosis Systems. An Introduction from Fault Detection to Fault Tolerance, Springer-Verlag, New York, NY.

Korbicz, J., Kościelny, J. M., Kowalczuk, Z. and Cholewa, W. (Eds.) (2004). Fault Diagnosis. Models, Artificial Intelligence, Applications, Springer-Verlag, Berlin/Heildelberg/New York, NY.

Korbicz, J. and Kościelny, J.M. (Eds.) (2010). Modelling, Diagnostics and Process Control. Implementation in the DiaSter System, Springer-Verlag, Berlin/Heildelberg.

Kościelny, J.M. (2001). Diagnostics of Automatized Industrial Processes, Academic Printing Office EXIT, Warsaw, (in Polish).

Kościelny, J.M. and Bartyś, M. (2000). Application of information system theory for actuator diagnosis, IFAC Symposium on Fault Detection, Supervision and Safety for Technical Processes, Budapest, Hungary, Vol. 2, pp. 949-954.

Kościelny, J.M. and Bartyś, M. (2003). Fuzzy logic application for diagnostic reasoning, 5th IFAC Symposium on Fault Detection, Supervision and Safety for Technical Processes SAFEPROCESS 2003, Washington, DC, USA, pp. 633-638.

Kościelny, J.M., Sędziak, D. and Zakroczymski, Z. (1999). Fuzzy-logic fault isolation in large-scale systems, International Journal of Applied Mathematics and Computer Science 9(3): 637-652.

Kościelny, J.M. and Syfert, M. (2006). Fuzzy diagnostic reasoning that takes into account the uncertainty of the faults-symptom relation, International Journal of Applied Mathematics and Computer Science 16(3): 27-35.
Patton, R., Frank, P. and Clark, R. (1989). Fault Diagnosis in Dynamic Systems. Theory and Applications, Prentice Hall, Engelwood Cliffs, NJ.

Patton, R., Frank, P. and Clark, R. (Eds.) (2000). Issues of Fault Diagnosis for Dynamic Systems, Springer-Verlag, Berlin/Heildelberg/New York, NY.

Syfert, M. (2006). The issue of diagnostic relation uncertainty and fault conditional isolability, Proceedings of 6th IFAC Symposium, SAFEPROCESS 2006, Beijing, China, Vol. 1, pp. 747-752.

Venkatasubramanian, V., Rengaswamy, R. and Kavuri, S.N. (2003). A review of process fault detection and diagnosis, Part II: Quantitative model based methods, Computers and Chemical Engineering 27(3): 293-311.

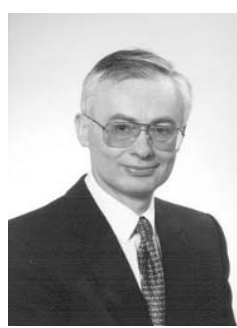

Michał Bartyś was born in Poznań, Poland, in 1949. He earned his M.Sc. degree at the Warsaw University of Technology in Poland in 1973 and the $\mathrm{Ph} . \mathrm{D}$. degree in mechanics at the same university in 1983. Since 1973 he has been working in the Institute of Automatic Control and Robotics, Warsaw University of Technology. In 1986-1988 he studied at Institut für Hydraulische und Pneumatische Antriebe und Steuerungen in RWTH Aachen in Germany. In 1993-1994 he visited Institut für Elektrotechnik und Automatisierungstechnik in Duisburg, Germany. His research activities are focused mainly on the fields of process control, fault detection and isolation, fault tolerant systems, fieldbus network systems, industrial applications of fuzzy logic, and development of intelligent automatic control components. He is a contributor of 9 books and 3 handbooks, an author of 131 papers in scientific journals and conferences, and an author or a co-author of 4 patents. He has invented 80 unique mechatronic devices.

Received: 16 December 2012

Revised: 21 March 2013 